\title{
Performance Evaluation of Fault Tolerance Protocols in MANET
}

\author{
Rohit Jain \\ PG scholar \\ Department of ECE \\ MIT, Mandsaur
}

\author{
Abhinav Mehta \\ PG scholar \\ Department of ECE \\ MIT, Mandsaur
}

\author{
Vinay Somani \\ Lecturer \\ Department of ECE \\ MIT, Mandsaur
}

\begin{abstract}
A mobile ad hoc network (MANET) is a collection of wireless mobile nodes communicating with each other using multi-hop wireless Links without any existing network infrastructure or centralized administration. It has been shown that using multiple paths to route messages between any sourcedestination pair of nodes (instead of using a single path) balances the load more evenly throughout the network. The common belief is that the same is true for ad hoc networks, i.e., multi-path routing balances the load significantly better than single-path routing. Our Protocol, called MPOLSR \& MDART is a multipath routing protocol for MANET. In addition route recovery \& loop detection are implemented in MPOLSR in order to improve quality of service regarding OLSR.MP-OLSR is suitable for mobile, large \& dense network with large traffic \& could satisfy critical multimedia applications with high on time constraints. While MDART is an efficient protocol which gives improved performance in large networks. MDART is an enhancement of shortest path routing protocol known as Dynamic Address Routing (DART).MDART discovers and stores multiple paths to the destination in the routing table. In this paper, we have compare and analysis the performance of proactive multipath routing protocols for MANET under different scenarios \& metrices using NS-2.
\end{abstract}

\section{Keywords}

MANET, MRP,MDART, MPOLSR, NS-2etc.

\section{INTRODUCTION}

There is a existence of another approach where mobile nodes utilizing each other as access points or relays for traffic when they cannot establish a direct communication with end points. That model of communication is called mobile Adhoc networks (MANETS). These networks can be set up randomly and when needed (on demand). In MANETS nodes have to announce their presence periodically and listen for their neighbor's announcements broadcasts to discover and learn how to reach each other. Therefore, mobility and scalability are the main challenges in the infrastructure less networks. Hence there is need for efficient routing protocols to allow the nodes to communicate over multihop paths,

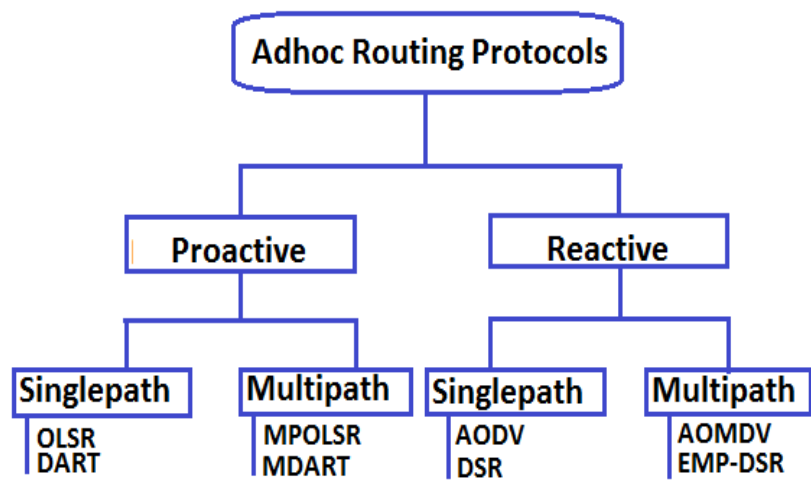

Consisting of several links, dynamic and non Predictable topology in a way that doesn't use any more of the network resources then necessary. Such factors can be for example the time taken for routes discovery or routing information update mechanism. In proactive routing, every host maintains at least one routing table to represent the whole topology of the network. The tables (of each host) are updated continuously. Therefore, routes are already available at any time some hosts want to communicate with each other. In order to maintain up-to-date routing information at all hosts, topology information has tube exchanged between all hosts on a regular basis. This increases the overhead in the network. On one hand, substantial bandwidth is used for the large control traffic; on the other hand, routes are always available in shortly for any communication request. This reduces the delays of data transmissions. One of the most important proactive protocols is the Optimized Link State Routing protocol (OLSR) unlike proactive routing protocols; reactive routing protocols initiate a route discovery process when needed. This reduces the overhead as compared to proactive routing protocols, but it increases the transmission delay. Another classification can be made according to number of paths a routing protocol delivers per source destination pair [7]. There exist unipath and multipath routing protocols. Unipath routing protocol: one route is used to deliver data from source node to destination node. Multipath routing protocol: more than one route is used to deliver the data.

\section{MULTIPATH ROUTING IN ADHOC NETWORKS}

Mobile ad hoc networks (MANETs) are characterized by a dynamic topology, limited channel bandwidth and limited power at the nodes. Because of these characteristics, paths connecting source nodes with destinations may be very unstable and go down at any time, making communication over ad hoc networks difficult. On the other hand, since all nodes in an ad hoc network can be connected dynamically in an arbitrary manner, it is usually possible to establish more 
than one path between a source and a destination. When this property of ad hoc networks is used in the routing process, we speak of multipath routing. In most cases (e.g.), the ability of creating multiple routes from a source to a destination is used to provide a backup route. When the primary route fails to deliver the packets in some way, the backup is used. This provides a better fault tolerance in the sense of faster and efficient recovery from route failures. Multiple paths can also provide load balancing and route failure protection by distributing traffic among a set of disjoint paths. Paths can be disjoint in two ways: (a) link-disjoint and (b) node-disjoint. Node-disjoint paths do not have any nodes in common, except the source and destination, hence the do not have any links in common. Link-disjoint paths, in contrast, do not have any links in common. They may, however, have one or more common nodes [5]

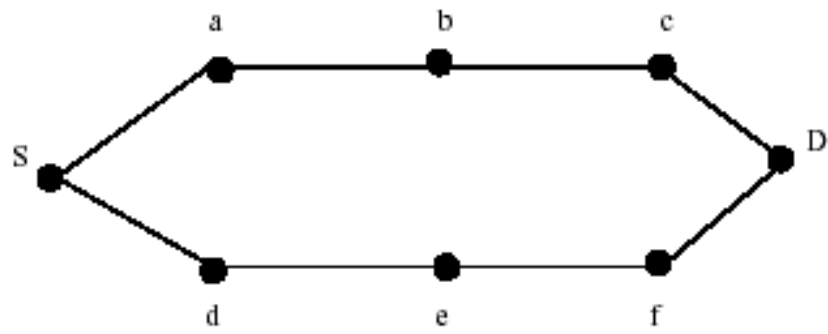

Fig 1. Two node-disjoint paths from source $S$ to destination $D$.

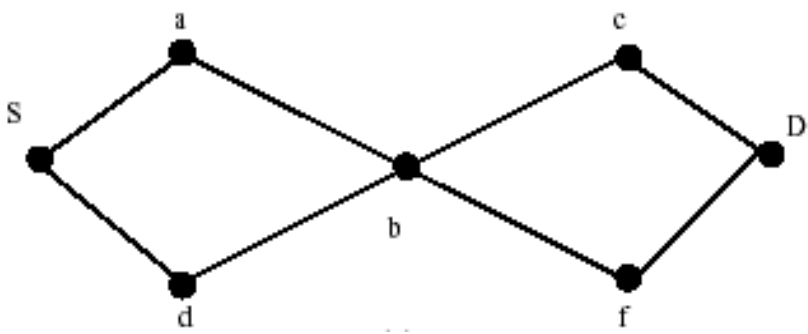

Fig 2.Two link-disjoint paths from source $S$ to destination $D$. Note that they are not node-disjoint, since they share node $b$.

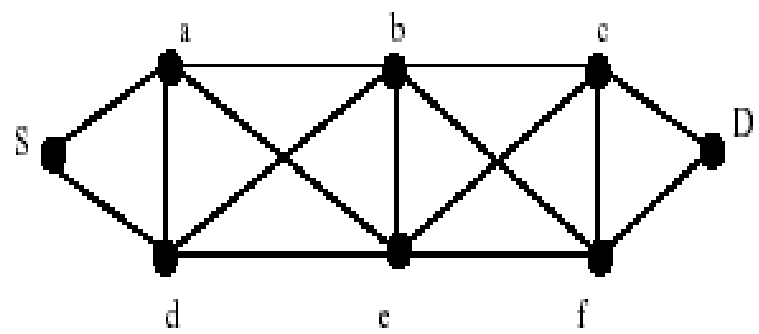

Fig 3.The two node-disjoint paths, when they are in each other's radio coverage.

In order to use multiple paths simultaneously they need to be as independent as possible. So not only do they need to be disjoint, also route coupling must be taken into account, because routes can interfere with each other. Route coupling takes place when a path crosses the radio coverage area of another path. There is a protocol that uses this property of radio broad cast to create backup-routes, but in the case of multiple-path data transport route coupling is unwanted. Routes may be link- or even node-disjoint but still interfere with each other due to route coupling. Consider the nodedisjoint routes of figure 1again. In the situation of figure 3 , when node $a$ for example sends data to node $\mathrm{b}$ (both route 1), node $d$ on the other route cannot transmit data to $e$ on route 2 ,since the nodes (and thus routes) are in each other's radio coverage area and interfere with each other. Since none of the routing protocols take the route coupling into account, we will ignore it in the sequel. Disjointness will be the only measure used for path independence.

\section{MDART}

M-DART shares several characteristics with DART. It is based on the distance vector concept and it uses the hop by hop routing approach. Moreover, M-DART also resorts to the dynamic addressing paradigm by using transient network addresses. The main difference between DART and M-DART lies in the number of routes stored in the routing table: the former stores no more than $l$ entries, one for each sibling, while the latter stores all the available routes toward each sibling. The core of M-DART protocol lies in ensuring that such an increase in the routing state information stored by each node does not introduce any further communication or coordination overhead by relying on the routing information already available in the DART protocol.

M-DART extends the DART protocol to discover multiple routes between the source and the destination. In such a way, M-DART is able to improve the tolerance of a tree-based address space against mobility as well as channel impairments. Moreover, the multi-path feature also improves the performances in case of static topologies thanks to the route diversity. M-DART has two novel aspects compared to other multi-path routing protocols [6-7]. First, the redundant routes discovered by M-DART are guaranteed to be communication-free and coordination-free, i.e., their discovering and announcing though the network does not require any additional communication or coordination overhead. Second, M-DART discovers all the available redundant paths between source and destination, not just a limited number.

In particular, it does not employ any special control Packet or extra field in the routing update entry and, moreover, the number of entries in the routing update packet is the same as DART: $l$. No special coordination action is needed by nodes and the node memory requirements constitute the only additional overhead in M-DART relative to DART.

\section{MPOLSR}

The MP-OLSR can be regarded as a kind of hybrid multipath Routing protocol which combines the proactive and periodically to detect the network topology, just like OLSR. However, MP-OLSR does not always keep a routing table. It only computes the multiple routes when data packets need to be sent out. The core functionality of MP-OLSR has two parts: topology sensing and route computation. The topology sensing is to make the nodes aware of the topology information of the network. This part benefits from MPRs like OLSR. The route computation uses the Multipath Dijkstra Algorithm to calculate the multipath based on the information Obtained from the topology sensing. The source route (all the hops from the source to the destination) is saved in the header of the data packets. The topology sensing and route computation make it possible to find multiple paths from source to destination. In the specification of the algorithm, the paths will be available and loop-free. However, in practice, the situation will be much more complicated due to the change of the topology and the instability of the wireless medium. So route recovery and loop detection are also proposed as auxiliary functionalities to improve the performance of the protocol[9]. The route recovery can effectively reduce the packet loss, and the loop detection can 
be used to avoid potential loop sin the network as depicted in we discuss both the core functionalities and auxiliary functionalities[6].

\section{METHODOLOGY}

\subsection{Simulation Environment}

Simulation environment is as follows:

Parameter Values

\begin{tabular}{|l|l|}
\hline Traffic type & CBR \\
\hline Simulation time & 600 seconds \\
\hline Maximum connections & $5,10,15,20$ \\
\hline Pause time & $0,100,200,300,400,500,600$ \\
\hline Area of the network & $1000 * 1000$ \\
\hline
\end{tabular}

\subsection{NS-2 (Network Simulator-2)}

The NS-2 [3] is a discrete event driven simulation and in this the physical activities are translated to events. Events in this are queued and processed in the order of their scheduled Occurrences. The functions of a Network Simulator [9] are to create the event scheduler, to create a network, for computing routes, to create connections, to create traffic. It is also useful for inserting errors and tracing can be done with it. Tracing packets on all links by the function trace-all and tracing packets on all links in nam +format using the function nam trace-all.

\subsection{Performance Metrics}

We report four performance metrics for the protocols:

Success Delivery Rate: It is the ratio of data received by the sink at the destination over the data sent by constraint bit rate source.

Throughput: Throughput is total packets successfully delivered to individual destination over total time divided by total time

Normalized Routing load: The Normalized routing loads measures by the total number of routing packets sent divided by the number of data packets delivered successfully.

Packet Loss: Packet loss occurs when one or more packets of data traveling across a network fail to reach their destination.

\section{SIMULATION RESULTS AND}

\section{ANALYSIS:}

We ran the simulation environments for $600 \mathrm{sec}$ for two scenarios with pause times varying from 0 to 600 second, maximum connections varying in between 5 to 20 connections.Success delivery rate, Throughput Normalized routing load \& Packet loss are calculated for MPOLSR and MDART. The results are analyzed below with their corresponding graphs.

\section{SUCCESS DELIVERY RATE:}

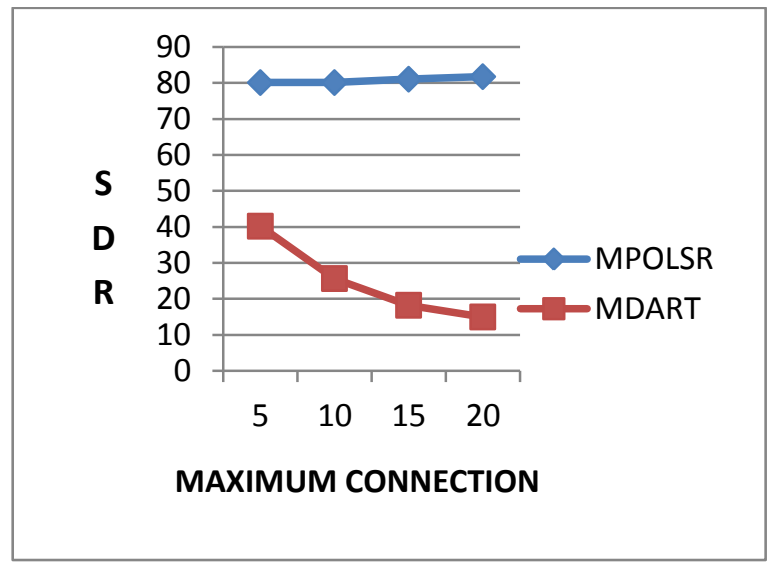

(A).Comparison of MPOLSR \& MDART on the basis of SDR with varying maximum connection, fixed nodes-50, traffic load-2(Pkts/sec), maximum Speed-20(m/s) \& Pause time-0(s).

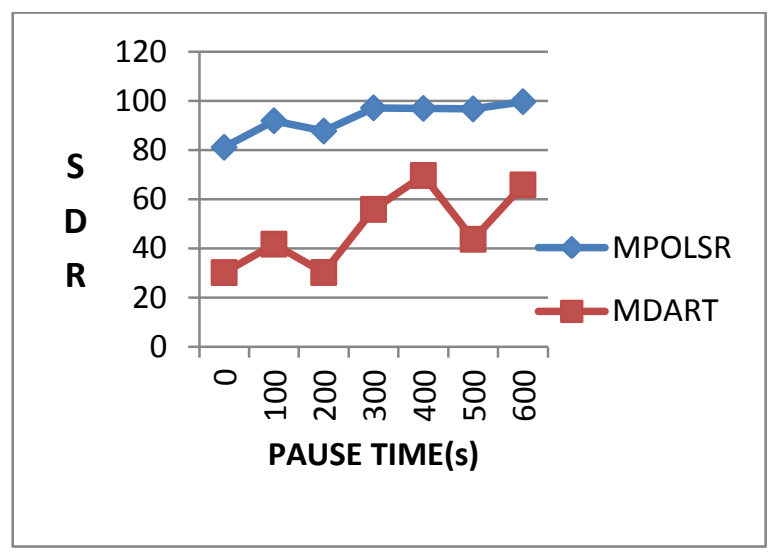

(B).Comparison of MPOLSR \& MDART on the basis of SDR with varying Pause time, fixed nodes-50, traffic load2(Pkts/sec), maximum Speed-20 $(\mathrm{m} / \mathrm{s})$.

Analysis of the Result: We note that in this simulation as in SDR, MPOLSR performs well by varying max.connection \& pause time(s) as compare to MDART.We also noticed that in both the protocols its value is exponentially increasing by increasing the value of parameters used in the scenarios.

\section{THROUGHPUT:}

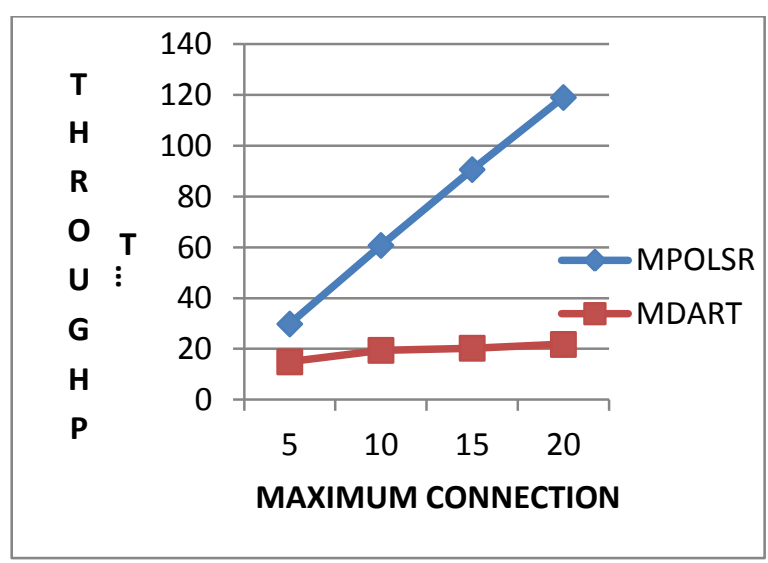

(A).Comparison of MPOLSR \& MDART on the basis of Throughput with varying maximum connection, fixed nodes50,traffic load-2, maximum Speed-20(m/s) \& Pause time-0(s). 


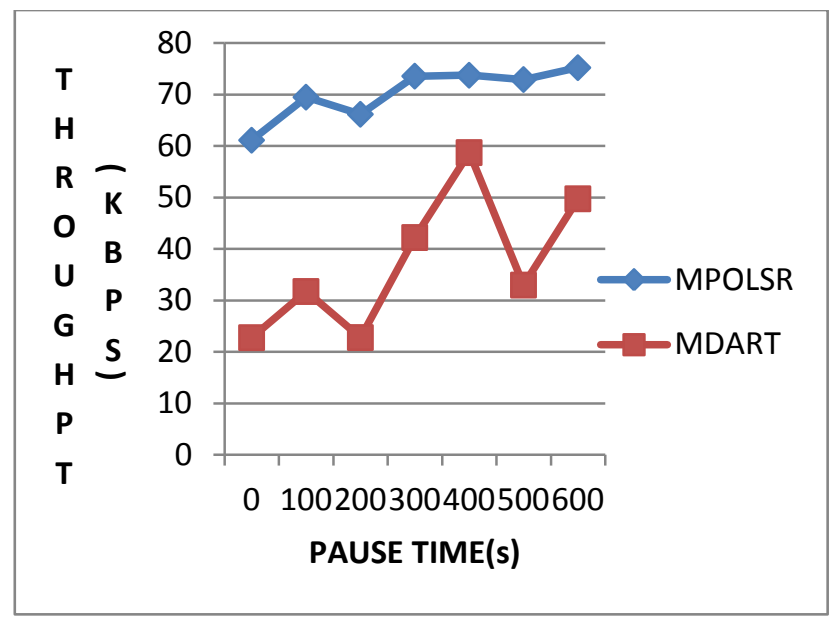

(B).Comparison of MPOLSR \& MDART on the basis of Throughput with varying pause time, fixed nodes-50, maximum connection, maximum speed-20(m/s) \& Pause time-0(s).

Analysis of the Result: We note that in this simulation as in Throughput, MPOLSR performs well in all cases by varying max.connection \& pause time(s) as compare to MDART.We also noticed that in both the protocols as in case of vary max. Connection its value is linearly increasing by increasing the value of parameters used in scenarios. But in case of varying pause time its value is exponentially increasing by increasing the value of parameters used in our scenarios.

\section{NORMALIZED ROUTING LOAD:}

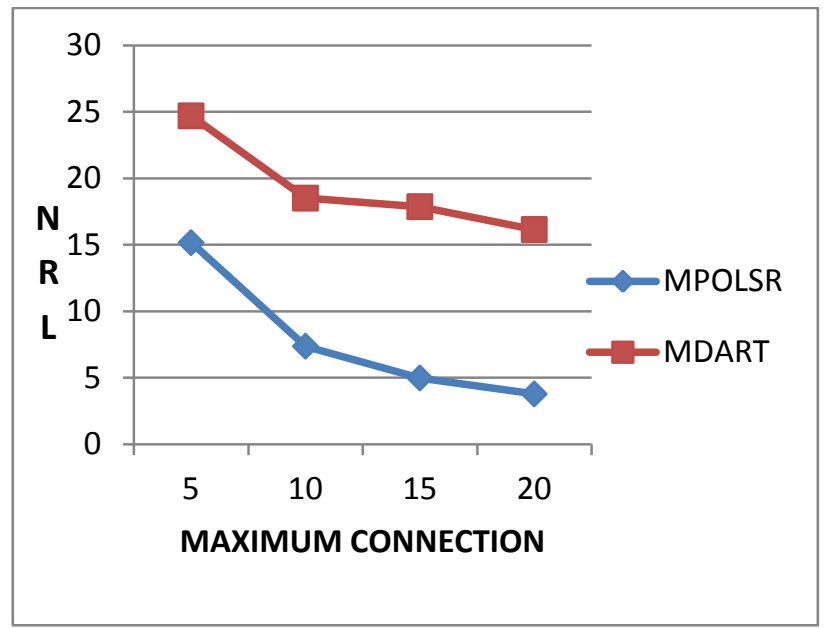

(A).Comparison of MPOLSR \& MDART on the basis of NRL with varying maximum connection, fixed nodes-50, traffic load-2, maximum speed-20 $(\mathrm{m} / \mathrm{s}) \&$ Pause time-0(s).

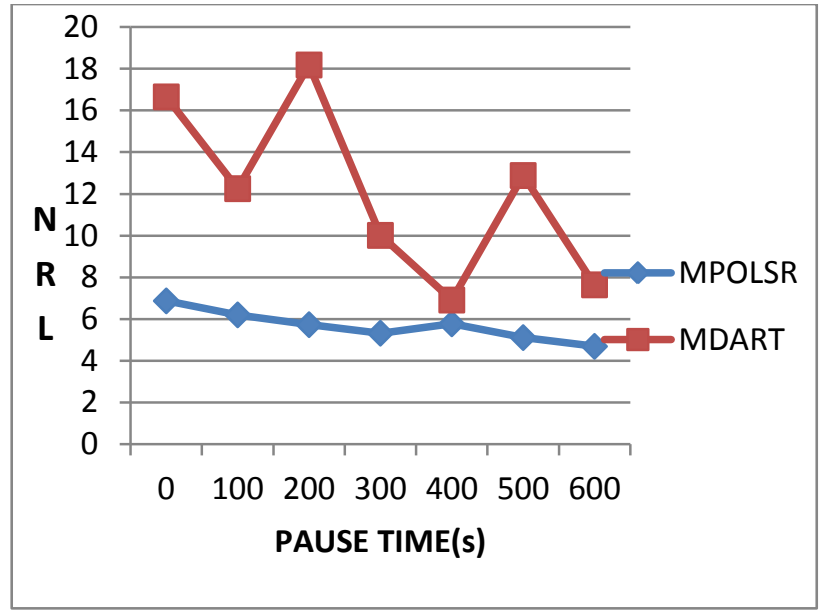

(B).Comparison of MPOLSR \& MDART on the basis of NRL with varying pause time, fixed nodes-50, traffic load-2, maximum connection-10 \& maximum speed-20 $(\mathrm{m} / \mathrm{s})$.

Analysis of the Result: We note that in this simulation as in NRL, MDART performs well in all cases by varying max.connection \& pause time(s) as compare to MPOLSR.We also noticed that in both the protocols as in case of varying max. connection its value is linearly decreasing by increasing the value of parameters used in scenarios. But in case of varying pause time its value is exponentially decreasing by increasing the value of parameters used in scenarios.

\section{PACKET LOSS:}

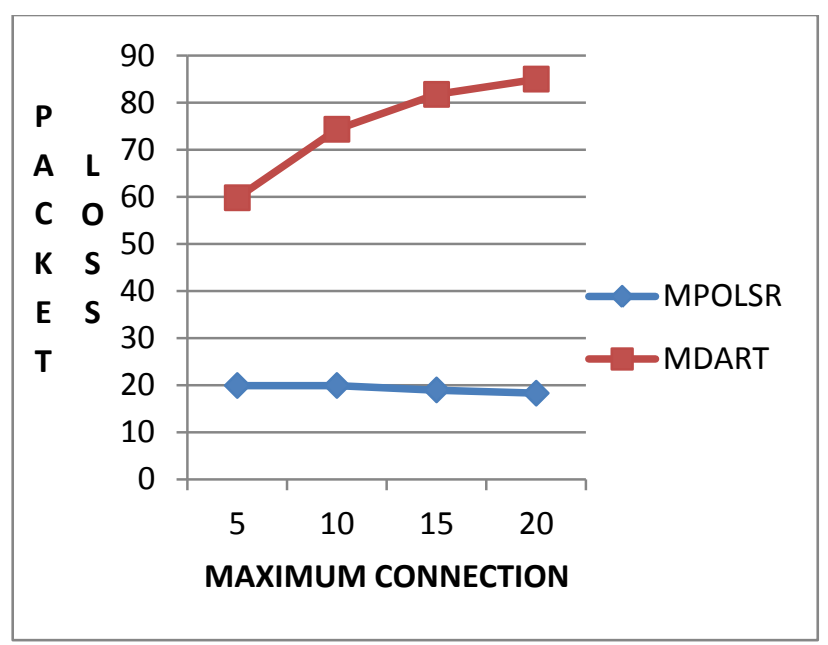

(A).Comparison of MPOLSR \& MDART on the basis of Packet loss with varying maximum connection, fixed nodes-50, traffic load-2, maximum speed-20(m/s) \& Pause time-0(s). 


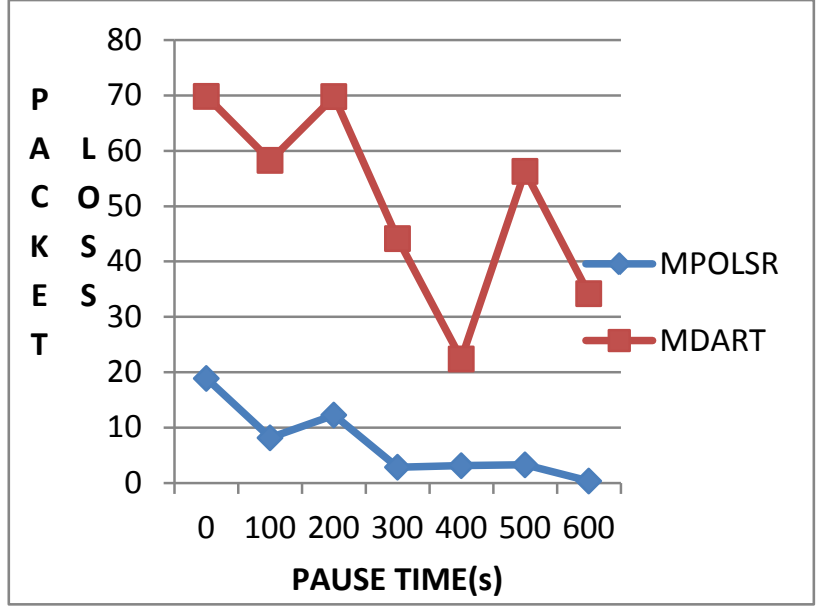

(B).Comparison of MPOLSR \& MDART on the basis of Packet loss with varying pause time, fixed nodes-50, traffic load-2, maximum connection \& maximum $\operatorname{speed}-20(\mathrm{~m} / \mathrm{s})$.

Analysis of the Result: We note that in this simulation as in Packet loss, MDART performance is more in all cases by varying max.connection \& pause time(s) as compare to MPOLSR.We also noticed that in both the protocols its value is linearly \& exponentially increasing by increasing the value of parameters used in the scenarios.

\section{CONCLUSION:}

This paper evaluated the performance of MPOLSR and MDART using NS-2.Comparison was based on the Success delivery rate, Throughput, Normalized Routing load \& Packet loss. We concluded that the performance of MPOLSR is better as compared to MDART in terms of SDR, Throughput by varying all the scenarios which is used in Simulation. In NRL \& Packet Loss Metrices, MDART performance is better as compared to MPOLSR by varying all the scenarios. As it is obvious that in one protocol if Success to delivery rate is high than packet loss is less \& Success to delivery rate is of low performance than packet loss is high. We also seen that as in both the protocols its value is lag, lead \& linearly increasing by increasing the value of parameters used in our simulation.

\section{REFERENCES}

[1]. M.K. Marina, S.R. Das, On-demand multi path distance vector routing in ad hoc networks, in Proceedings of the Ninth International Conference on Network Protocols, IEEE Computer Society, Washington, DC, USA, 2001, pp. 14-23.
[2]. S.Lee, M. Gerla, Split multipath routing with maximally disjoint paths in ad hoc networks, Helsinki, Finland, 2001, pp. 3201-3205.

[3]. M. Caleffi and L. Paura, "M-DART: Multi-path Dynamic Address Routing", Wirel. Commun. Mob. Comput. pp: 1-20, 2010.

[4]. H. Badis, K.A. Agha, Qolsr multi-path routing for mobile Adhoc networks based on multiple metrics: bandwidth and delay, in: IEEE Vehicular Technology Conference, Los Angeles, CA, USA, 2004, pp.2181-2184.

[5]. M. Kun, Y. Jingdong, R. Zhi, The research and simulation of multipath OLSR for mobile ad hoc network, in: International Symposium on Communications and Information Technologies (ISCIT), 2005, pp.540-543.

[6]. J. Eriksson, M. Faloutsos and S. Krishnamurthy. "DART: Dynamic Address Routing for Scalable Ad Hoc and Mesh Networks". in IEEE- ACM Transactions on Networking, vol. 15, no. 1, April 2007, pp. 119-132.

[7]. M.Caleffi, G.Ferraiuolo and L.Pauro, "On Reliability of Dynamic Addressing Protocols in Mobile Ad hoc Networks", Proceedings of WRECOM'07, Wireless Rural and Emergency Communications Conference, Roma, Italy, October 2007.

[8].R.Krishan and J.A. Silvester. Choice of allocation granularity in multipath source routing schemes. In IEEE INFOCOM'99, volume 1, pages322-329, IEEE, 1993.

[9]. C.S.R. Murthy and B.S. Manoj, Ad Hoc Wireless Networks: Architecture and Protocols, ch. Routing Protocols for Ad hoc Wireless Network, pp. 299.

[10].A Nasipuri and S.R. Das. On-demand multi-path routing for mobile adhoc networks. In IEEE ICCCN'99, pages 64-70, IEEE, 1999.

[11]M. Tarique, K.E. Tepe, S. Adibi, S. Erfani, Survey of multipath routing Protocols for mobile ad hoc networks, Journal of Network and Computer Applications 32 (2009) 1125-1143.

[12]T. Clausen, C. Dearlove, IETF Request for Comments: 5497, Representing Multi-Value Time in Mobile Ad Hoc Networks (MANETs), March 2009.

[13].T. Clausen and P. Jacquet "Optimized Link State Routing Protocol (OLSR).” RFC 3626, IETF Network Working Group, October 2003. 